\title{
Flow Injection Analysis with Simple Chemiluminescence Detector for Hypoxanthine
}

\author{
Kaoru Yagiuda, Akihide Hemmi, Kenji SAKAMURA, Satoshi ITO, Yasukazu ASANO*, \\ Kenji $\mathrm{HAYASHI}^{\dagger}$ and Isao $\mathrm{KARUBE}^{\dagger \dagger}$
}

Received June 30, 1995; Accepted September 10, 1995

\begin{abstract}
A low cost chemiluminescence detector with a photodiode for flow injection analysis (FIA) of hypoxanthine in fish and meat was developed for quality control in food industry. In this system, hypoxanthine is oxidized enzymatically to produce hydrogen peroxide during passage through the xanthine oxidase immobilized reactor. Then, chemi-luminescence caused by mixing hydrogen peroxide with chemiluminescence reagent was detected by the flow through photodiode cell that was designed to detect efficiently weak chemiluminescence. This light intensity was proportional to hypoxanthine concentration. As a result, a linear working curve was obtained from $2 \times 10^{-7}$ to $2 \times 10^{-4} \mathrm{~mol} \mathrm{l}^{-1}$ hypoxanthine concentration in this FIA system. The present system with the photodiode detector was applied to the determination of hypoxanthine in fish and meat samples. The results were compared with those obtained by the conventional HPLC method. Analytical results for fish samples obtained by the present FIA method were fairly in good agreement with those obtained by the conventional HPLC method. Correlation factor and regression line between both methods were 0.978 and $Y=0.89 X+0.66$ respectively. The present FIA system was concluded to be available as a simple, easy-handling and reliable instrument for quality control in food industry.
\end{abstract}

\section{INTRODUCTION}

Hypoxanthine is produced by decomposition of ATP is a chemical index of freshness and aging of food such as fish and meat.

A $\mathrm{K}$ value is an important factor which decides fish freshness ${ }^{1}$. There have been some reports on the analytical methods for hypoxanthine such as HPLC method $^{21}$ with an amperometric detection ${ }^{3,4)}$, an enzyme sensor ${ }^{5}$ and FIA system with amperometric biosensor ${ }^{6.7)}$. In the previous paper ${ }^{(0.7)}$, we found out that a 2 channel FIA system with the enzyme sensor for free amino acid and hypoxanthine was useful for the quality control of beef during aging which can monitor the progress of aging as well as taste of beef.

Biosensing with optical transducers has been DKK CORPORATION (4-13-14 kitamachi, Kichijoji, Musashino, Tokyo, 180 Japan)

†NICHIREI CORPORATION (6-19.20 Chuo-ku, Tsukiji, Tokyo, 104 Japan)

$t \leftarrow$ Research Center for Advanced Science and Technology, The University of Tokyo (4-6-1 Komaba Meguro-ku Tokyo, 153 Japan) Key Words: Flow Injection Analysis, Chemiluminescence Detector, Enzyme Column, Hypoxanthine growing its interest because of high sensitivity, ease of operation and insensitivity to electrical disturbance ${ }^{8,9,10,111}$. Chemiluminescence reaction of luminol with hydrogen peroxide has been well known as one of the high sensitive analytical technique to detect many chemical substances ${ }^{12}$. However, up to now, a expensive luminometer with the photo-multiplier has been used to detect chemiluminescence sensitivity. On the other hand, the careful quality control for the products in the industrial field has been getting to be more important by effect of ISO-9001 for the analytical instruments and HACCP for the foods ${ }^{13)}$. The establishment of products reliability has been needed urgently. At the present time, the manual analysis by skillful experts of chemical analysis was mainly employed in quality control in the products. Especially, quality control of taste of foods has been done by sensing test of the experts. Therefore the development of a low cost, simple, easy-handling and reliable analytical instrument at line for quality control has been demanded.

Biosensor and biosensing systems using a photodiode were proposed for on line process monitoring and 
control in industrial field mentioned above as a trial to application for a biosensor ${ }^{14,15)}$. However, there have been few studied on the application of the photodiode to detection of FIA for the determination of hypoxanthine in fish and meat.

In order to construct a simple, stable, high sensitive, low cost and reliable FIA system for the determination of hypoxanthine, we have developed a FIA system with a photodiode detector and an enzyme column using an enhanced chemiluminescence reaction of $\mathrm{H}_{2} \mathrm{O}_{2}$ with piodephenol which was proposed by Thorpe ${ }^{1(1)}$. An utilization of the photodiode as the detector for the FIA system was enable to reduce the cost of FIA.

The chemiluminescence detector was specially designed for detecting weak light of chemiluminescence efficiently and applied it for hypoxanthine analysis in fish and meat samples. In the FIA system, hypoxanthine is oxidized by the catalytic reaction of xanthine oxidase immobilized on the column to generate hydrogen peroxide. Then, chemiluminescence based on luminol reaction by mixing hydrogen peroxide with chemiluminescence reagents is detected by the photodiode detector. The reaction relations are as follows.

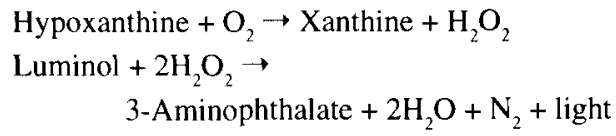

\section{EXPERIMENTAL}

\section{1 Reagents}

All chemicals used were of analytical reagent grade. Xanthine oxidase from butter milk and peroxidase from horseradish peroxidase (HPR) were obtained from Sigma Chemical Company. Chemiluminescence reagent was prepared by mixing luminol, $\mathrm{p}$-iodephenol and HPR with a $0.2 \mathrm{~mol} \mathrm{l}^{-1}$ carbonate buffer (pH9.40). The optimum concentration of the above component in the chmiluminescence reagent was $10^{-5} \mathrm{~mol} \mathrm{l}^{-1}, 2 \times 10^{-5}$ mol $1^{-1}$ and $2 \times 10^{-3}$ mol $1^{-1}$ respectively. Pure water treated with a $0.45 \mu \mathrm{m}$ membrane filter, which its electric conductivity is less than $0.1 \mu \mathrm{S} / \mathrm{cm}$, was used through the work. A $0.1 \mathrm{~mol} \mathrm{I}^{-1}$ phoshate buffer ( $\mathrm{pH}$ 7.8) was used as a carrier solution. The chemiluminescence for reagents were freshly prepared just before use for every experiments.

\section{2 Preparation of xanthine oxidase immobilized} column

A xanthine oxidase immobilized column was prepared by the following procedures.

(1) The porous silica-beads, aminopropyl CPG (CPG Inc.) which has pore size $500 \AA$ and mesh size $200 / 400$, was packed into a column $(3 \mathrm{~mm}$ I.D., $18 \mathrm{~mm}$ long).

(2) A $0.1 \mathrm{~mol} \mathrm{l}^{-1}$ carbonate solution ( $\mathrm{pH} \mathrm{8.5)} \mathrm{was}$ circulated for 30 minutes with a peristaltic pump into the column.

(3) A 4\% glutaraldehyde solution was circulated for 90 minutes as the same manner as above.

(4) After these procedures, the column was washed with pure water by the same procedure for 2 hours.

(5) A solution of xanthine oxidase (5 units a column) was circulated into the column for 2 hours to immobilized the enzyme onto the porous silica beads.

(6) After circulating a $0.1 \mathrm{~mol} \mathrm{1}^{-1}$ glycine solution into the column for 2 hours, the column was filled with a $0.1 \mathrm{~mol} \|^{-1}$ phosphate buffer solution ( $\mathrm{pH}$ 7.80 ).

(7) Thus the prepared xanthine oxidase immobilized column fulled with the phosphate buffer solution was stored in a refrigerator at $4^{\circ} \mathrm{C}$.

\section{3 Fabrication of photodiode detector}

The detector was fabricated by assembling a $\mathrm{Si}$ photodiode (Hamamatsu Photonics Co.Ltd., S12271010 type, size : $10 \times 10 \mathrm{~mm}$ ) which photosensitivity is $0.42 \mathrm{~A} / \mathrm{W}$ at $720 \mathrm{~nm}$, into the SUS 316-stainless steel body (Its size: 30 height $\times 30$ width $\times 20$ depth) as shown in Fig. 1. The photodiode was powered with the $\pm 15 \mathrm{~V}$ regulated DC power supply. The detector has a large cell window area $(10 \mathrm{~m} \emptyset \times 1 \mathrm{~mm}$ thickness $)$ and surface of the window faced to the photodiode was polished to work as concave lens to detect chemi-

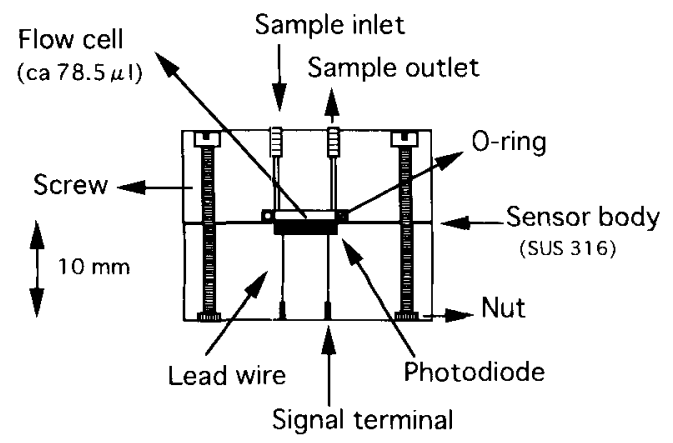

Fig. 1 Structure of chemiluminescence detector. 
luminescence light effectively. The flow cell was set as the distance of $0.9 \mathrm{~mm}$ far from the photodiode. The volume of the flow cell was ca $78.5 \mu 1$.

\section{4 FIA system with photodiode detector}

The two channel FIA system consisted of a pump (Masterflex Co.Ltd., PA-21A type), an injector (Rheodyne Co. Ltd., 7125 type), a xanthine oxidase immobilized column, a photodiode detector, an amplifier (Hamamatsu Photonics Co.Ltd., S-2719 type) and a recorder (Sekonic Co.Ltd. 250-F type) as shown in Fig. 2. The flow rate of the pump was $2 \mathrm{ml} / \mathrm{min}$. The $10 \mathrm{~cm}$-long PTFE tube $(0.5 \mathrm{~mm}$ I.D.) from the mixing point of the luminol reagent and a sample to the detector was decided experimentally as the longer tube made sensitivity to chemiluminescence lower. For the first time, the system was calibrated by injecting the standard hypoxanthine solution into the buffer stream. After that, a $100 \mu \mathrm{l}$ of sample was injected into the buffer stream, and the evolved hydrogen peroxide by enzyme reaction in the column was transferred to merge with the luminol reagent. Light caused by chemiluminescence reaction was monitored by the detector located downstream of the flow system. The light intensity was amplified by the amplifier and its signal was fed to the recorder. The concentration of hypoxanthine was determined by the change of the detector output which was observed as a peak-shaped signal on the recorder. The FIA system was calibrated at constant intervals with the standard hypoxanthine injection to prevent the measurement errors from the minor change of the luminol-regents.

\section{5 Analytical sample preparation}

One gram stored at $2^{\circ} \mathrm{C}$ of the fish and meat samples were immersed and extracted into $100 \mathrm{ml}$ of $10 \%$ perchloric acid for $30 \mathrm{~min}$ then the extracts were diluted with pure water at hypoxanthine concentration of $2 \times 10^{-6}$ to $10^{-4} \mathrm{~mol} \mathrm{l}^{-1}$ after neutralizing with $\mathrm{KOH}$ solution the extracts and eliminating perchlorate ion as potassium perchlorate from them because of interference from perchlorate for the highly sensitive detection of hypoxanthine by the photodiode detector.

\section{RESULTS AND DISCUSSION}

\section{1 Optimum conditions of FIA system}

Optimum conditions of chemiluminescence reaction in this system were set up after investigations concerning $\mathrm{pH}$, flow rate of the carrier solution, concentration of $\mathrm{p}$ iode phenol, luminol, HRP and sample volume using hydrogen peroxide of $10^{-7}$ to $10^{-4} \mathrm{~mol}^{-1}$ referring to the previous study ${ }^{15)}$. The result based on the pretests was described in 2.1 and 2.4. A linear calibration curve from $2 \times 10^{-7}$ to $2 \times 10^{-4} \mathrm{~mol}^{-1}$ for hydrogen peroxide was obtained under the optimum conditions of this system. It means that hydrogen peroxide more than $10^{-7}$ mol $1^{-1}$ is needed to evolve by the enzyme reaction for chemiluminescence in this system. Intensity of chemiluminescence for the $10^{-2} \mathrm{~mol}^{-1}$ hypoxanthine solution was about 30 times higher compared with that without of p-iode phenol in the luminol regent. In this pretests, it was found that flow rates of the regent and carrier solutions was very important for the sensitive determination of hypoxanthine because that decom-

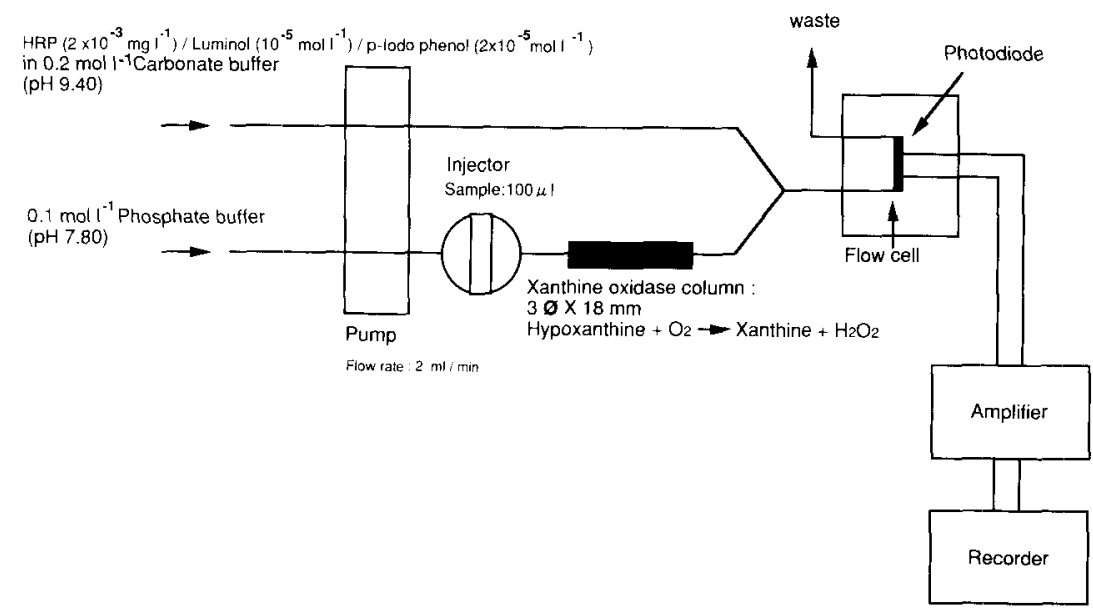

Fig. 2 Flow diagram of chemiluminescence flow injection analysis system for determination of hypoxanthine. 
position and diffusion of hypoxanthine took place in the column.

\section{2 Calibration curve}

We prepared several standard solutions of hypoxanthine and investigated the relationship between the concentration and the peak height based on FIA signal according to analytical conditions as shown in Fig. 2 which were decided experimentally based on the previous- reports ${ }^{13)}$. A linear calibration curve was obtained from $2 \times 10^{-7}$ to $2 \times 10^{-4} \mathrm{~mol} \mathrm{l}^{-1}$ of hypoxanthine. This shows that conversion of hypoxanthine to hydrogen peroxide by xanthine oxidase proceeds efficiently in the column. The lower detection limit was $10^{-7} \mathrm{~mol}^{-1}$ for hypoxanthine. However, in case that concentration of hypoxanthine was more than $2 \times$ $10^{-4} \mathrm{~mol} \mathrm{l}^{-1}$ chemi-luminescence was reduced suffering from effect of uric acid which oxidation by xanthine oxidase was progressed. The repeatability of FIA signals was $0.25 \%$ of the standard deviation for 5 times injections of the same samples at $10^{-6}$ mol $1^{-1}$ hypoxanthine. Figure 3 shows the typical FIA signals of five tests for the same concentration of hypoxanthine in this FIA system. The sampling rate was about $60 \mathrm{hr}^{-1}$. This indicates that the photodiode detector could be available as a new, simple and sensitive detector of FIA for hypoxanthine. The change of baseline with FIA system was about less than $0.24 \%$ to the peak height at the measurement of $10^{-6} \mathrm{~mol} \mathrm{l}^{-1}$ hypoxanthine. The

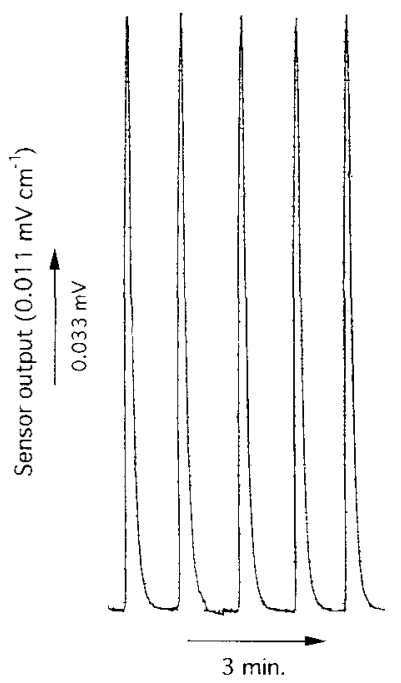

Chart speed $\left(\mathrm{min} . \mathrm{cm}^{-1}\right)$

Fig. 3 Typical FIA signals for hypoxantine.

Hypoxantine : $10^{-6} \mathrm{~mol} \mathrm{l}^{-1}$ current signal from the photodiode converted to potential with current-voltage convertor and the peak height based on potential was proportional to hypoxanthine concentration. A slope of the calibration curve in the range of $2 \times 10^{-7}$ to $2 \times 10^{-4} \mathrm{~mol} \mathrm{~J}^{-1}$ hypoxanthine was $2.95 \times 10^{-2} \mathrm{mV} / \mathrm{mol} \mathrm{l}^{-1}$.

\section{3 Stability of xanthine oxidase immobilized} column

The long term stability of the column immobilized with xanthine oxidase was investigated. The column store at $4^{\circ} \mathrm{C}$ was tested periodically for a $10^{-6} \mathrm{~mol} \mathrm{I}^{-1}$ hypoxanthine sample. After 1 month, the output signal from the detector decreased about $20 \%$ compared with the initial value. The column performance was checked at the same concentration of hypoxanthine every 2 week. The signal from the detector was stable within $\pm 10 \%$ relative errors for five months running. The column performance for one year showed that activity was decreased by $50 \%$ of the intial value. Theses mean that the column with immobilized xanthine oxidase was durable to use for one year at least.

\section{4 Selectivity}

The specificity for hypoxanthine of the present FIA system using the photodiode detector and the column with immobilized xanthine oxidase was studied for several substances which ofter coexist in foods samples. Peak heights for the sample containing hypoxanthine of $10^{-4} \mathrm{~mol} \mathrm{I}^{-1}$ were compared with solution containing $10^{-3}$ mol $\mathrm{l}^{-1}$ of substances such as L-glutamic acid, Llactate, glucose, pyruvic acid, inosine 5'-monophosphate, inosine, guanosine and guanine. No detector signal was observed for above substance. This indicates that above substances did not interfere the determination of hypoxanthine because the enzyme reaction by xanthine oxidase was specific to hypoxanthine.

However, output of the detector for a xanthine solution at $10^{-4}$ mol $1^{-1}$ showed $54 \%$ of that for the hypoxanthine solution of the same concentration. This means that xanthine is also oxidized similarly to hypoxanthine by xanthine oxidase.

\section{5 Correlation between the present FIA method and conventional HPLC method for fish samples}

We applied the present FIA system for the determination of hypoxanthine in 13 fish samples. The analytical results obtained by the present method were compared with that by the conventional HPLC method with UV detector. The validation of HPLC method was 
shown in Table 1. The correlation of analytical results between two methods is fairly well as shown in Fig. 4. From Fig. 4, we obtained the regression line expressed by $\mathrm{Y}=0.89 \mathrm{X}+0.66$ and a correlation factor of 0.978 . This indicates that the present FIA system with the photodiode detector can be applied for the determination of hypoxanthine in food sample.

Table 1 Analytical conditions of HPLC method for hypoxanthine.

\begin{tabular}{|c|c|}
\hline Items & Analytical conditions \\
\hline Instrument & Shimadzu LC-6A \\
\hline Eluate & $0.05 \mathrm{M}$ phosphate buffer \\
\hline Column & 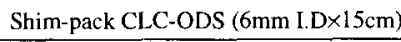 \\
\hline Flow rate & $0.7(15 \mathrm{~min}) \mathrm{ml} / \mathrm{min}$ \\
\hline Column temperature & $40^{\circ} \mathrm{C}$ \\
\hline Measurement pressure & 150 atom $/ \mathrm{cm}^{2}$ \\
\hline Wave length & UV $254 \mathrm{~nm}$ \\
\hline Sample volume & $20 \mu \mathrm{I}$ \\
\hline
\end{tabular}

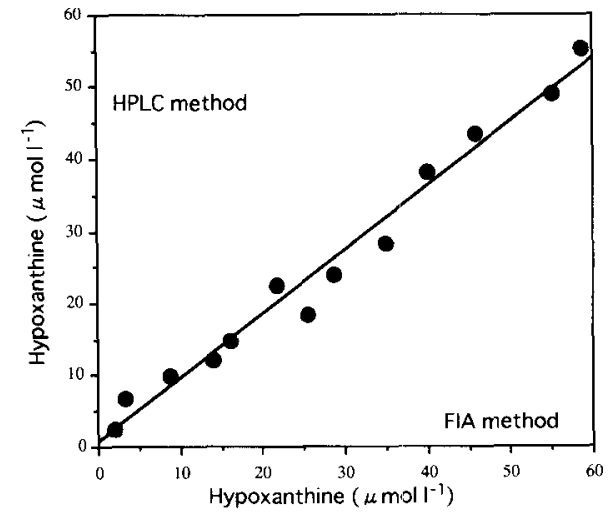

Fig. 4 Correlation between HPLC method and FIA method for determination of hypoxanthine.

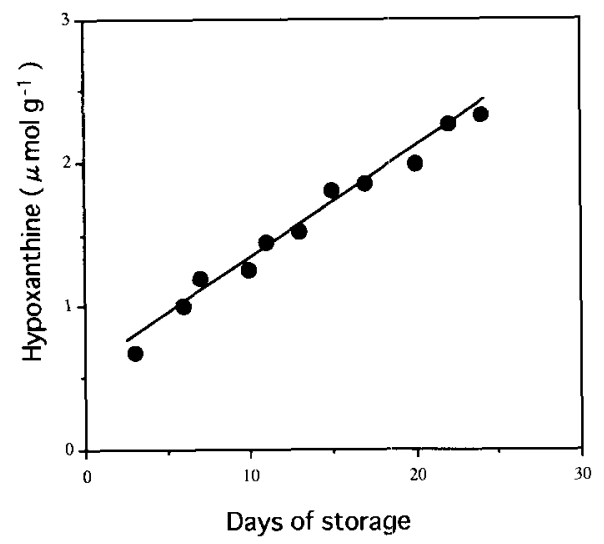

Fig. 5 Changes in amounts of hypoxanthine in meat stored at $2^{\circ} \mathrm{C}$.

\section{6 Changes in amounts of hypoxanthine in meat samples}

We applied the present FIA system to monitoring the time aging course of the meat sample. The specimens of sirloin meat stored at $2^{\circ} \mathrm{C}$ were used for the experiments at regular time intervals. As the result was shown in Fig. 5, the amount of hypoxanthine in the meat sample almost increased linearly with the storage period. From these data, it is clear that hypoxanthine was good index for evaluating the lapse of aging time.

\section{CONCLUSION}

We have developed the FIA system with the photodiode detector for the determination of hypoxanthine in fish and meat samples. A linear cali-bration curve was obtained from $2 \times 10^{-7}$ to $2 \times 10^{-4} \mathrm{~mol}^{-1}$ of hypoxanthine. This method was applied to determination of hypoxanthine in fish and meat samples. Correlation factor and regression line between the present method and conventional HPLC method for 13 fish samples were 0.978 and $\mathrm{Y}=0.89 \mathrm{X}+0.66$ respectively. And also, it was clear that this FIA system was useful for judging the state of aging in meat.

We conclude that the present FIA system has advantage of simplicity, low cost, easy maintenance and applicability as the quality control technique for the determination of hypoxanthine at the manufacturing process in the food industry. The base-line of FIA signal was very stable. However, it was needed to calibrate the system pediorically because the luminol regents were not stable for long term. Therefore, it was expected to stabilize the luminol reagents from the view of quality control at line in food industry.

The basic concept described in this paper may be also applicable to evaluate other determining substances in food samples.

\section{References}

1) Y. Nakatani, T. Fujita, S. Sawa, T. Otani and I. Takagahara, Agric. Biol. Chem., 50, 1751 (1986).

2) M. Yoshiura, T. Iwamoto and K. Iriyama, Jikeikai Med. $J ., 33,37$ (1986).

3) T. Yao, Y. Kobayashi and S. Musha, Anal. Chim. Acta, 138, 81 (!982).

4) H. Okuma, T. Takahashi, S. Sekimukai, K. Kawahara and R. Akahoshi, Anal. Chim. Acta, 244, 161 (1991).

5) E. Watanabe, K. Ando, I. Karube, H. Matsuoka and 
S. Suzuki, J. Food Sci., 48, 496 (1983).

6) Y. Yano and Y. Asano. JAPANESE TECHNOLOGY REVIEWS (ON-LINE SENSORS FOR FOOD PROCESSING), Vol. 4, No. 2, 36-43 (1994).

7) M. Hishimoto, M. Kanaya, K. Hayashi, T. Okugawa, Y. Asano, T. Youdou and N. Funazaki, "Food Industry and Sensors", Korin Publishing Co. Ltd., 323 (1991).

8) T. M. Freeman and W. R. Seiz, Anal. Chem., 50, 1242 (1978).

9) M. Aizawa, Y. Ikariyama and H. Kuno, Anal. Lett., 17 (B7), 555 (1984).

10) Garry H. G. Thorpe, Larry J. Kricka, Susan B. Moseley and Thomas P. Whitehead, Clinical Chemistry, Vol. 31, No. 8, 1335-1334 (1985).
11) L. J. Blum, S. Gautier and P. R. Coulet, Anal. Lett., 21 137 (1988).

12) H. D. Albrecht, Z. Phys. Chem., 136, 321 (1928).

13) ICMSF, 1988 : Microorganisms in Foods 4. Application of Hazard Analysis Critical Point (HACCP) system to Ensure Microbiological Safety and Quality. London, Blackwell Scientific Publications.

14) F. Preuschoff, U. Spohn and D. Janasek, Biosensors \& Bioelectronics, 9, 543 (1994).

15) K. Hayash E. Tamiya and I. Karube, 59th Spring Annual Meeting, Abstract I, The Chemical Society of Japan, 160 (1990).

16) Garry H. G. Thorpe and Larry J. Kricka, Methods in Enzymology, Academic Press Inc. 133, 331 (1986). 